\title{
Supraglottic airway devices for surfactant treatment: systematic review and meta-analysis
}

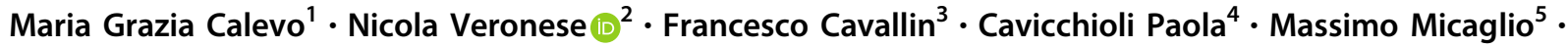 \\ Daniele Trevisanuto ${ }^{6}$
}

Received: 29 June 2018 / Revised: 26 September 2018 / Accepted: 24 October 2018 / Published online: 5 December 2018

(c) Springer Nature America, Inc. 2018

\begin{abstract}
Objective To compare surfactant administration via supraglottic airway device (SAD) vs. nasal CPAP alone or INSURE. Study design A systematic search of PubMed, EMBASE, SCOPUS, Cochrane Central Register of Controlled Trials and Clinicaltrials.gov was performed. Articles meeting inclusion criteria (RCT, surfactant administration via SAD, laryngeal mask, I-gel) were assessed

Results Five RCTs were eligible. Surfactant administration via SAD reduced the need for intubation/mechanical ventilation (RR 0.57, 95\%CI 0.38-0.85) and short-term oxygen requirements (MD -8.00, 95\%CI - 11.09 to -4.91) compared to nCPAP alone. Surfactant administration via SAD reduced the need for intubation/mechanical ventilation (RR 0.43 , 95\%CI 0.31-0.61), but increased short-term oxygen requirements (MD 3.10, 95\%CI 0.51-5.69) compared to INSURE approach. Conclusions In preterm infants with RDS, surfactant administration via SAD reduces the need for intubation/mechanical ventilation. Overall, available literature includes few, small, poor-quality studies. Surfactant administration via SAD should be limited to clinical trials.
\end{abstract}

\section{Introduction}

Respiratory distress syndrome (RDS), caused by surfactant deficiency, is the most frequent neonatal condition in preterm infants $[1,2]$. Surfactant treatment has been extensively studied in preterm infants and has been shown to significantly decrease air leaks and neonatal mortality [3-6]. In 1994, Verder et al. showed that the INSURE (INtubation, SURfactant delivery using an endotracheal

Daniele Trevisanuto

daniele.trevisanuto@unipd.it

1 Epidemiology, Biostatistics and Committees Unit, Istituto Giannina Gaslini, Genoa, Italy

2 National Research Council, Neuroscience Institute, Aging Branch, Padua, Italy

3 Independent statistician, Solagna, Italy

4 Pediatric Department, Ospedale dell'Angelo, Mestre, Venice, Italy

5 Department of Anesthesia and Intensive Care, Careggi University Hospital, Florence, Italy

6 Department of Woman's and Child's Health, University of Padova, Padova, Italy tube and Extubation after a brief period of mechanical ventilation) approach was effective in reducing the need for subsequent mechanical ventilation in preterm infants with moderate-to-severe RDS receiving nasal continuous positive airway pressure (nCPAP) [7]. Premedication with opioids are used during this procedure [8].

Endotracheal intubation is an extremely distressing, painful procedure and has the potential for airway injury; in addition, it may be associated with significant hemodynamic instability including hypoxia, bradycardia, blood pressure fluctuations and increased intracranial pressure [9, 10].

Recently, new methods of modified INSURE without using an endotracheal tube are increasingly being used around the world. These techniques, known as less invasive surfactant administration (LISA) or minimally invasive surfactant treatment (MIST), refer to surfactant administration through a thin feeding tube or a small angiocatheter positioned in the trachea while the patient is breathing spontaneously and receiving noninvasive respiratory support [11-14]. Unfortunately, as happens with the INSURE approach, LISA or MIST techniques also require laryngoscopy, limiting their advantage to the avoidance of mechanical ventilation during surfactant delivery. 
Surfactant administration via a supraglottic airway device (SAD) has been initially described in few, small studies showing feasibility and safety in preterm infants $[15,16]$. The reported advantages of SAD over endotracheal tubes comprise a reduced invasiveness because the airway is not instrumented with a laryngoscope and easier positioning $[17,18]$. These benefits suggest that surfactant administration via SAD may be a very promising approach for the treatment of preterm infants with RDS [19].

The objective of this systematic review and meta-analysis of randomized controlled trials (RCTs) was to compare surfactant administration via SAD under two alternative scenarios: (1) continued nasal CPAP without surfactant administration and (2) surfactant administration via endotracheal tube (INSURE) in terms of need for intubation and/ or mechanical ventilation, oxygen requirements and major neonatal outcomes in preterm infants with RDS.

\section{Materials and methods}

\section{Search strategy and selection of studies}

To identify relevant studies, we systematically searched MEDLINE/PubMed, EMBASE, SCOPUS, Cochrane Central Register of Controlled Trials and Clinicaltrials.gov, as well as the abstracts of the annual Pediatric Academic Societies meetings from 1990. We also hand-searched the reference lists of retrieved articles to identify additional studies of interest. The search strategy was carried out without language restrictions from database inception until 31 December 2017. Two researchers (MM and PC) independently reviewed the search results and screened the titles and abstracts. We obtained the full texts of all potentially eligible studies. In PubMed, the following search strategy was used: (Laryngeal mask) OR (supraglottic airway device) OR (intubation) AND (surfactant) AND (respiratory distress syndrome) AND (newborn OR neonatal OR neonate OR neonates OR "Low Birth Weight" OR "Small for Gestational Age" OR prematur* OR preterm OR Postmature OR infant* OR birth). This search strategy was adapted to suit the other electronic sources. Conference abstracts and reference lists of included articles were handsearched to identify any potential additional relevant articles. Data were checked and any inconsistencies were resolved by consensus with a third author (DT).

\section{Criteria for considering studies for this review}

Randomized controlled trials (RCTs) comparing nCPAP plus surfactant delivery via LMA vs nCPAP without surfactant administration and nCPAP plus surfactant via LMA vs n-CPAP plus surfactant via ETT were considered eligible for this review. Inclusion criteria for this metaanalysis were: (i) RCT design; (ii) preterm and term infants with a radiograph and clinical presentation consistent with RDS; (iii) surfactant administration via SAD. Studies not including humans were excluded. No language restriction was placed on the included studies.

Data collection extraction For each article, we extracted all specified outcome measures and the following additional data: title, first author, journal, year of publication, country, type of surfactant used in the treated group, number of participants, age, gender, mode of delivery, birth weight, gestational age, and the use of antenatal steroids. When queries arose or additional data were required, we contacted the trial authors.

Outcomes We included as primary outcomes:

1. intubation and/or mechanical ventilation $(\mathrm{Y} / \mathrm{N})$

2. oxygen supplementation from the time of administration of surfactant (expressed as $\mathrm{FiO} 2$ at 1-6 h), (mean and SD)

3. oxygen supplementation from the time of administration of surfactant (expressed as $\mathrm{FiO} 2$ at $12-24 \mathrm{~h}$ ) (mean and SD)

We considered as secondary outcomes:

1. number of surfactant doses, (mean and SD)

2. administration of $\geq 2$ surfactant doses, $(\mathrm{Y} / \mathrm{N})$

3. pulmonary air leak, $(\mathrm{Y} / \mathrm{N})$

4. intraventricular hemorrhage, grade III or IV (IVH) based on Levine classification, (Y/N)

5. bronchopulmonary dysplasia (BPD), defined as dependence on $\mathrm{O}_{2}$ or any ventilator support at the later of 28 days of age or 36 weeks postmenstrual age, $(\mathrm{Y} / \mathrm{N})$

6. mortality before discharge, $(\mathrm{Y} / \mathrm{N})$

7. length of hospital stay, (mean and SD)

8. bradycardia $(<90 \mathrm{bpm})$ during the procedure, $(\mathrm{Y} / \mathrm{N})$

9. serious adverse events (including severe desaturation, emergent intubation, pulmonary hemorrhage, prolonged bradycardia, or apnea) $(\mathrm{Y} / \mathrm{N})$

10. surfactant reflux and/or gastric residuals, $(\mathrm{Y} / \mathrm{N})$

Assessment of risk of bias Two authors (MGC, NV) independently appraised the risk of bias of the included studies using the Cochrane Collaboration's tool through seven aspects of potential bias; random sequence generation, allocation concealment, blinding of participants and personnel, blinding of outcome assessment, incomplete outcome data, selective reporting, and other bias (including 
early termination and absence of prospective sample size calculation) [20]. The risk of bias was categorized as high, low, unclear.

Quality of evidence We used the Grading of Recommendations Assessment, Development, and Evaluation (GRADE) approach, as outlined in the GRADE Handbook $[21,22]$, to assess the quality of evidence for the above outcomes. Two authors (MGC, FC) independently assessed the quality of evidence for each outcome. We considered evidence from RCTs as high quality, but downgraded evidence one level for serious (or two levels for very serious) limitations on the basis of the following: design (risk of bias), consistency across studies, directness of evidence, precision of estimates, and presence of publication bias. We used the GRADEpro GDT Guideline Development Tool to create a "Summary of findings" table to report the quality of evidence [23]. As described, this approach yields an assessment of the quality of a body of evidence according to one of four grades: high, moderate, low and very low. The:high" rating indicates that the true effect lies close to the estimate of the effect, while the "very low" grade indicates that the true effect is likely substantially different from the estimate.

SAD used in the studies The SAD used in the studies included classic LMA (3 studies) [24-26], ProSeal LMA (1 study) [27] and I-gel (1 study) [28]. (Fig. 1) The classic LMA, made of medical grade silicone, consists of an oval shaped mask with an inflatable outer rim; a wide bore airway tube, which originates from the back plate, is joined at the proximal end with a $15 \mathrm{~mm}$ standard connector (Teleflex Medical Europe Ltd, Westmeath, Ireland). The ProSeal LMA is a type of LMA with modified features (larger and deeper bowl, enlarged and softer mask, gastric drainage tube) potentially resulting in a better seal with periglottic

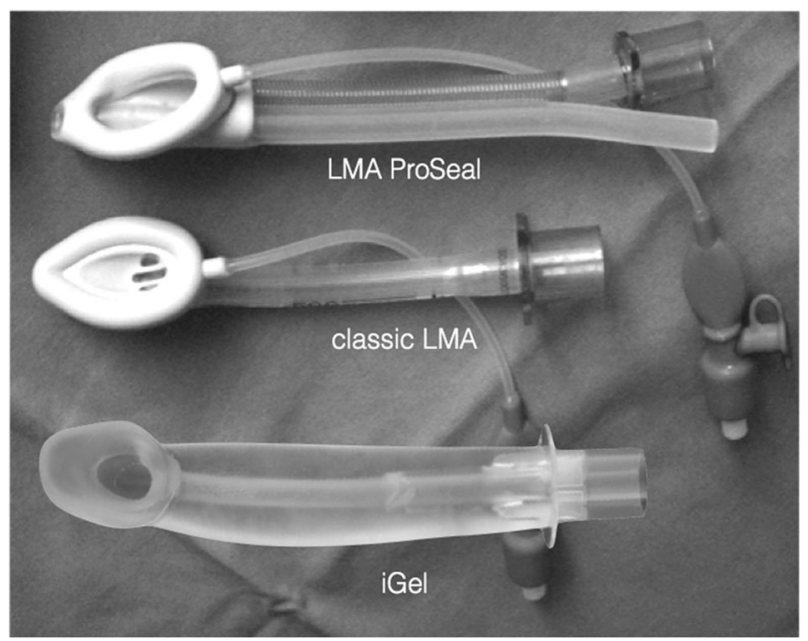

Fig. 1 Supraglottic airway devices used in the studies tissues (Teleflex Medical Europe Ltd, Westmeath, Ireland). The I-gel is a disposable SAD whose cuff, made from medical grade thermoplastic elastomer gel, does not require inflation with air to provide an efficient seal (Intersurgical Ltd, Berkshire, UK).

Data synthesis and statistical analysis Review Manager version 5.3 software was used to perform statistical analysis [29]. The standard methods of the Cochrane Collaboration were used to synthesize the data. For categorical data we calculated relative risk (RR). Mean and standard deviation (SD) were obtained for continuous data and analysis performed using mean difference (MD) and weighted mean difference when appropriate. For each measure of the effect the 95 per cent confidence intervals (CIs) were calculated. We combined the trials using the fixed-effect model. For estimates of RR and RD, we used the Mantel-Haenszel method. For all outcome measures, we assessed the statistical heterogeneity between trials using the $I^{2} . I^{2}>50 \%$ was interpreted as significant heterogeneity.

\section{Results}

\section{Search results}

Altogether, the searches yielded 575 non-duplicated articles. After excluding 518 articles based on title/abstract, 57 articles were retrieved for full text review. Finally, five RCTs were included in the qualitative and quantitative synthesis [24-28]. (Fig. 2)

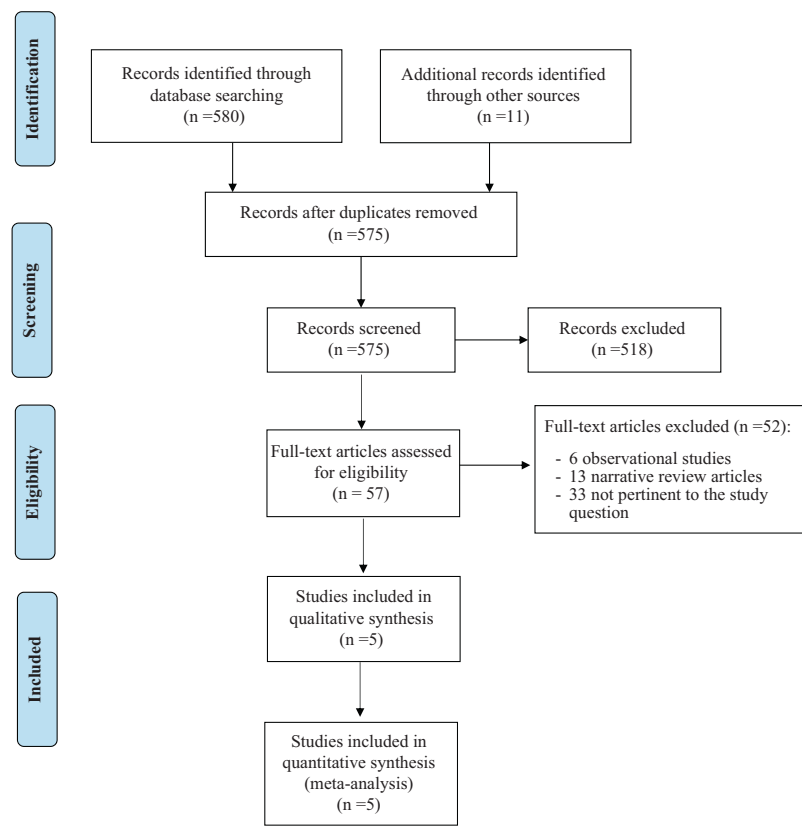

Fig. 2 PRISMA flow-chart 


\section{Study and patient characteristics}

Five trials recruiting 307 newborns (154 in intervention groups, 153 in control groups) met the inclusion criteria [24-28] (Table 1). Four studies were conducted in North [24-26], or South America [27], and one in Asia (Iran) [28]. Two RCT recruiting 129 newborns (63 in intervention groups, 66 in control groups) compared nCPAP plus surfactant delivery via LMA vs nCPAP without surfactant administration [24, 26]. Three studies recruiting 178 newborns (91 in intervention groups, 87 in control groups) were randomized controlled trials comparing nCPAP plus surfactant via LMA vs nCPAP plus surfactant via endotracheal tube $[25,27,28]$

\section{Risk of bias in included studies}

As reported in Fig. 3, all included RCTs were at high risk of bias, mainly due to the lack of blinding of health care providers and outcome assessors. One trial had high risk of selection bias: mode for random sequence generation was not reported and did not use opaque envelopes [28]. In Barbosa et al., the risk of selection bias was low regarding random sequence generation, but high regarding allocation concealment [27]. In the other three trials, the risk of selection bias was low as regards random sequence generation and allocation concealment [24-26]. Owing to the nature of the intervention, all trials were unblinded, leading to high risk of performance bias. In addition, no trials blinded researchers assessing trial endpoints of study treatments. Most trials accounted for all outcomes [24-27]. In Sadeghnia et al., this information was not clear [28]. Three trials provided complete results for all reported outcomes [24, 25, 27]. The primary outcome was changed in Attridge et al. [26] and the study protocol was not available in Sadeghnia et al. [28]. In Attridge et al., an early termination of the trial was decided due to slow enrollment [26]. Projected sample size was for 183 infants, but after 33 months a total of only 26 subjects had been enrolled. In Barbosa et al., the sample size was calculated as 30 patients per group [27]. An interim analysis, after 48 patients were enrolled and randomized, showed that both groups were equivalent. Therefore, due to ethical reasons, it was decided to interrupt the study since the primary outcome was achieved with 26 patients in LMA group. In Pinheiro et al., patient enrollment was stopped (not predefined in the protocol) after 60 patients instead of the 88 originally planned [25]. In Roberts et al., the study was stopped after 4 years due to slow enrollment, 103 out of 144 planned patients had been enrolled [24]. Sardeghnia et al. did not report the sample size calculation [28]. The quality of the evidence supporting the pooled outcome measures was very low according to the GRADE guidelines, due to limitations in the study design, early termination in some studies and imprecision of the estimates (few studies; few events).

\section{Effects of intervention}

\section{nCPAP plus surfactant delivery via LMA vs nCPAP without surfactant administration}

Meta-analysis of the pooled outcome measures is shown in Table 2.

Primary outcome measures Two trials $(n=129)$ reported data on the progression to intubation and/or mechanical ventilation $[24,26]$. Meta-analysis showed a significant reduction of the number of infants needing intubation and/or mechanical ventilation in nCPAP plus surfactant groups (RR $0.57,95 \%$ CI 0.38 to 0.85 ; participants $=129$ ). (Fig. 4)

As shown in Table 2, only Roberts et al. reported the $\mathrm{FiO} 2$ at $1-6 \mathrm{~h}$ after surfactant administration as mean and SD [24]. Surfactant administration via SAD was more effective than CPAP without surfactant in improving $\mathrm{FiO} 2$ at $1-6 \mathrm{~h}$ (MD $-8.00,95 \% \mathrm{CI}-11.09$ to -4.91 ; participants =103). (Fig. 5) In Attridge et al., median FiO2 at $1 \mathrm{~h}$ after surfactant administration was significantly lower in the LMA group than in controls: 0.25 (IQR 0.21-0.27) vs. 0.37 (IQR 0.34-0.49) ( $p=0.002)$ [26]. In Roberts et al., no significant differences emerged in terms of $\mathrm{FiO} 2$ at $12-24 \mathrm{~h}$ (MD $-1.00,95 \% \mathrm{CI}-5.28-3.28$; participants =103) [24]. However, in Attridge et al., median $\mathrm{FiO} 2$ at $12 \mathrm{~h}$ from the surfactant administration remained significantly lower in the LMA group than in controls: 0.27 (IQR 0.24-0.36) vs. 0.40 (IQR 0.31-0.45) in 26 participants ( $p=0.04$ as reported by authors) [26].

Secondary outcome measures Only Roberts et al. reported data on the number of surfactant doses [24]; LMA group was associated with increased number of surfactant doses $($ MD 0.70, 95\% CI 0.35-1.05; participants = 103) $($ data obtained by authors) and the need for $\geq 2$ doses (RR 2.01, 95\% CI 1.04-3.90; participants $=103$ ) than the group nCPAP without surfactant.

Meta-analysis showed no significant difference in pulmonary/air leak and bronchopulmonary dysplasia. (Table 2) Only Roberts et al. reported data on intraventricular hemorrhage and mortality (no events) [24]. No serious adverse events were reported in either study [24, 26]. In Roberts et al., 9 out of $50(18 \%)$ subjects who received surfactant via LMA had $>50 \%$ of the administered dose aspirated from the stomach [24]. In Attridge et al., one infant $(7.7 \%)$ in the LMA group had $>25 \%$ of the surfactant volume in the stomach contents [26]. Length of hospital stay was not reported in either study. 


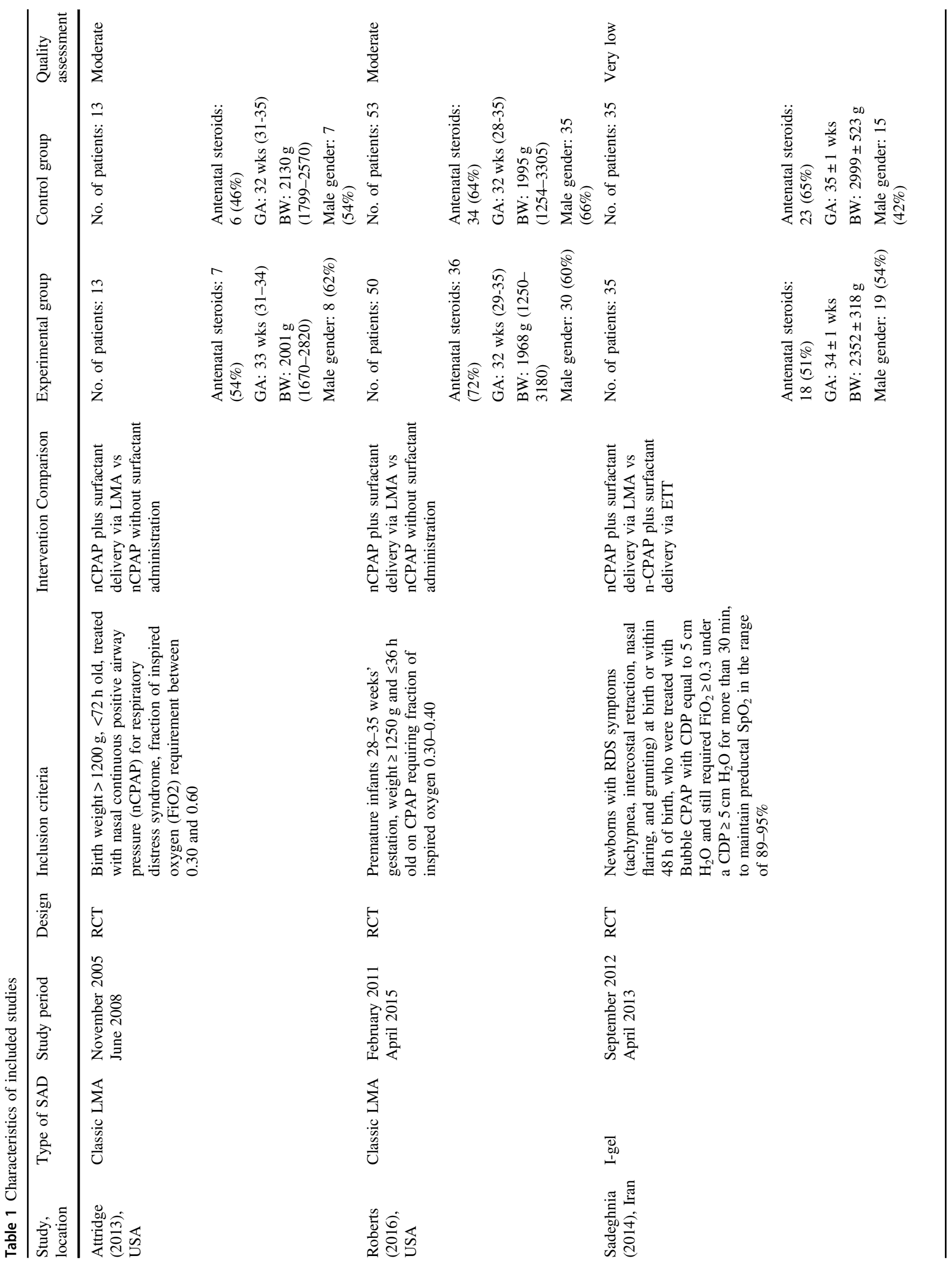




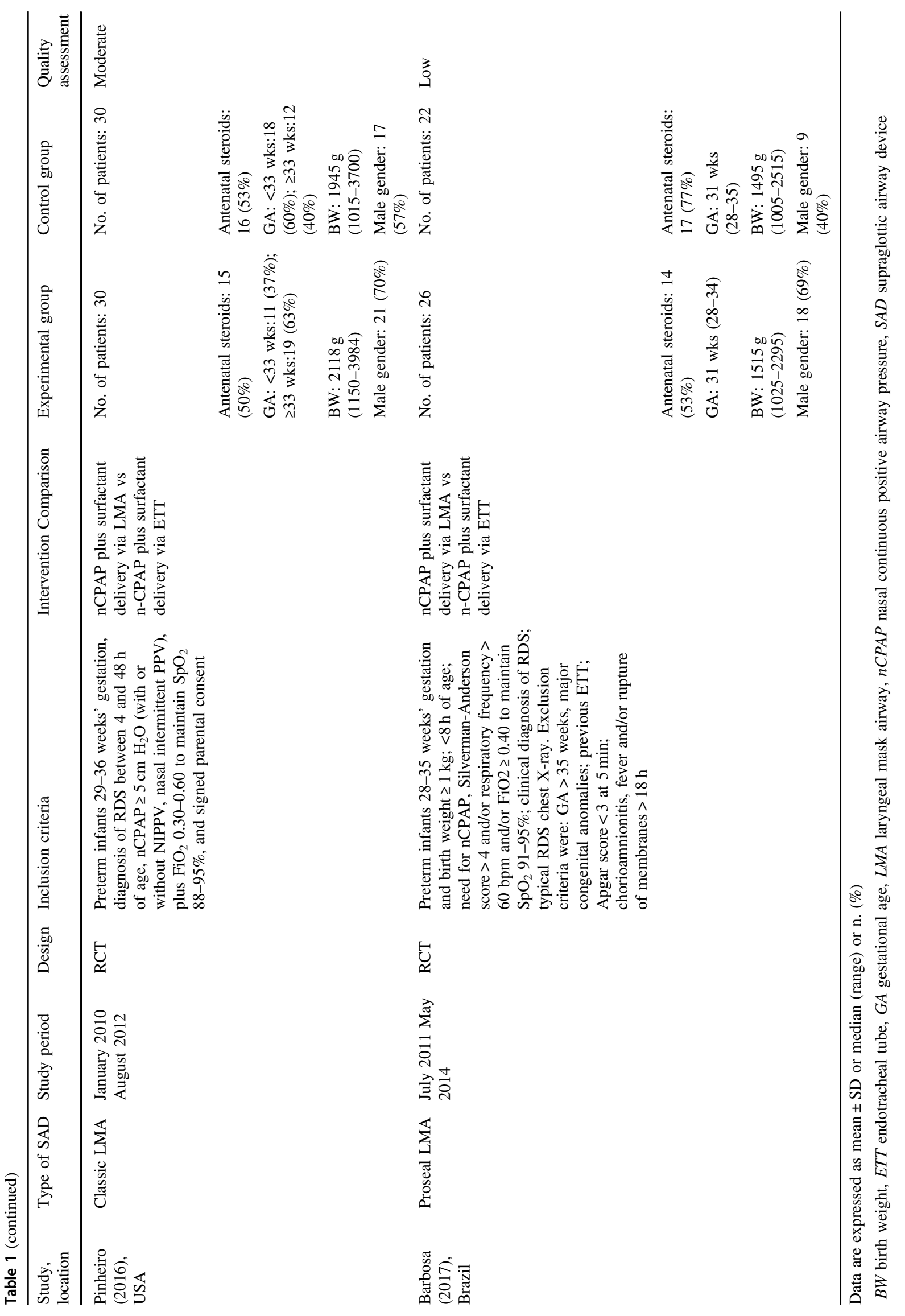




\section{nCPAP plus surfactant delivery via LMA vs $n$-CPAP plus surfactant via ETT}

Meta-analysis of the pooled outcome measures is shown in Table 3.

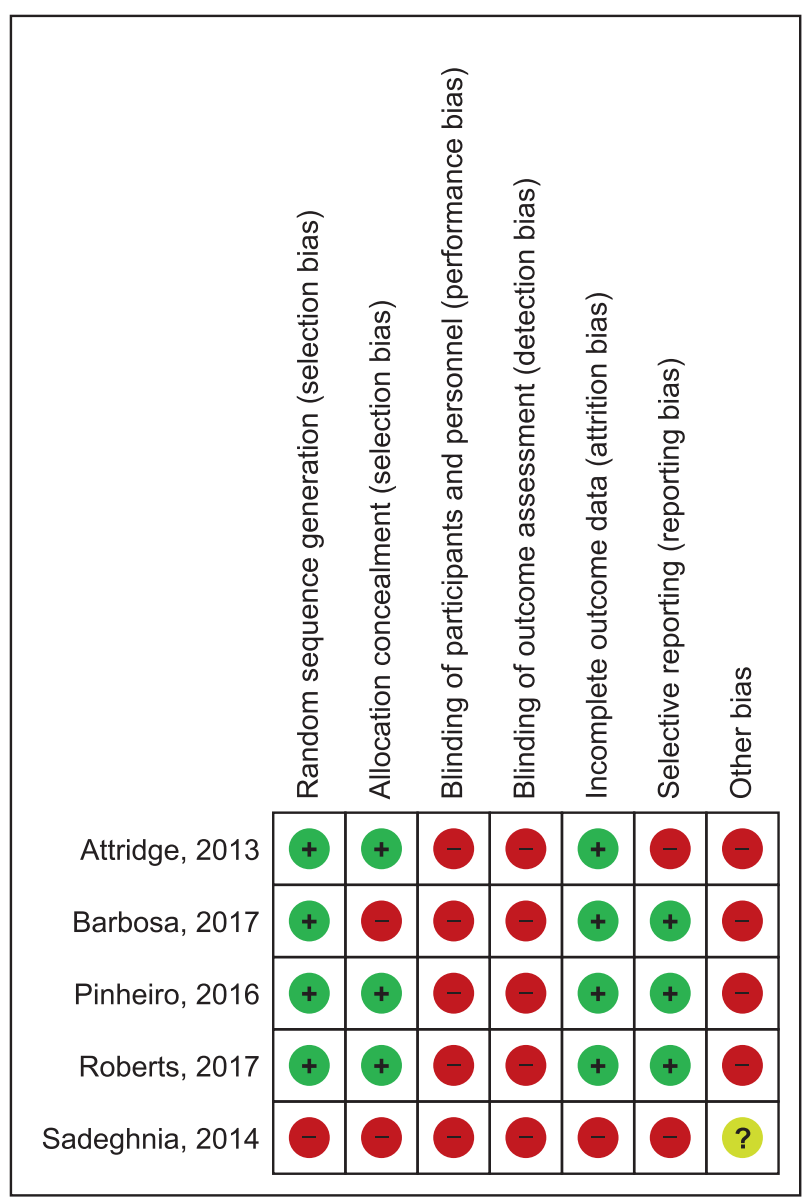

Fig. 3 Risk of bias summary
Primary outcome measures In all three studies, progression to intubation and/or mechanical ventilation was reported as an outcome measure [25, 27, 28]. Sadeghnia et al. reported no events in either group [28]. The metaanalysis showed a significant reduction in the need for intubation and/or mechanical ventilation in the interventional group (RR $0.43,95 \%$ CI 0.31 to 0.61 ; participants $=$ 178). (Fig. 6) Surfactant administration via SAD was less effective than surfactant via ETT in improving $\mathrm{FiO} 2$ at 1-6h (MD 3.1, 95\% CI 0.51-5.69; participants 108) (Fig. 7), while no significant difference was observed at 12-24 h (MD 3.00, 95\% CI -2.59-8.59; participants $=60$ ). (data obtained by authors)

Secondary outcome measures Only Pinheiro et al. reported data on the number of surfactant doses [25]; the LMA group was associated with increased number of surfactant doses (MD 0.60, 95\% CI 0.08-1.12; participants $=60$ ). There were no differences between the groups treated with surfactant via LMA or via ETT in terms of need for $\geq 2$ doses of surfactant (RR 1.02, 95\% CI 0.44-2.36; studies = 3 ; participants $=178$ ).

Occurrence of pneumothorax/pulmonary air leak (RR $1.63,95 \%$ CI $0.55-4.83$; studies $=3$; participants $=178$ ) and bronchopulmonary dysplasia (RR 3.05, 95\% CI $0.87-$ 10.75 ; studies $=2$; participants $=108$ ) were not different between the two groups. Only one study reported data on intraventricular hemorrhage: $5(19.2 \%)$ events occurred in LMA group and $3(13.6 \%)$ in the ETT group (RR 1.41, 95\% CI 0.38-5.25; participants $=48$ ) [27]. No difference in terms of mortality was observed between the groups (RR $0.17,95 \%$ CI 0.01 to 3.37 ; studies $=2$; participants $=$ 108). Length of hospital was similar between the groups receiving surfactant via LMA or via ETT (RR -3.88, $95 \% \mathrm{CI}-11.06$ to 3.29 ; participants $=108)$. (data obtained by authors)

Table 2 Data and analysis: nCPAP plus surfactant delivery via LMA vs nCPAP without surfactant administration

\begin{tabular}{|c|c|c|c|c|}
\hline Outcome & No. of studies & Participants & Statistical method & Effect estimate \\
\hline \multicolumn{5}{|l|}{ Primary outcomes } \\
\hline Need for intubation and/or mechanical ventilation & 2 & 129 & Risk ratio $(\mathrm{M}-\mathrm{H}$, Fixed, $95 \% \mathrm{CI})$ & $0.57[0.38,0.85]$ \\
\hline $\mathrm{FiO} 2$ at $1-6 \mathrm{~h}$ after surfactant administration & 1 & 103 & Mean difference (IV, Fixed, 95\% CI) & $-8.00[-11.09,-4.91]$ \\
\hline $\mathrm{FiO} 2$ at $12-24 \mathrm{~h}$ after surfactant administration & 1 & 103 & Mean difference (IV, Fixed, 95\% CI) & $-1.00[-5.28,3.28]$ \\
\hline \multicolumn{5}{|l|}{ Secondary outcomes } \\
\hline Number of surfactant doses & 1 & 103 & Mean difference (IV, Fixed, 95\% CI) & $0.70[0.35,1.05]$ \\
\hline Need $\geq 2$ doses of surfactant & 1 & 103 & Risk ratio $(\mathrm{M}-\mathrm{H}$, Fixed, 95\% CI) & $2.01[1.04,3.90]$ \\
\hline Pulmonary air leak & 2 & 129 & Risk ratio (M-H, Fixed, 95\% CI) & $0.65[0.23,1.88]$ \\
\hline Intraventricular hemorrhage (grade 3/4) & 1 & 103 & Risk ratio (M-H, Fixed, 95\% CI) & Not estimable \\
\hline Bronchopulmonary dysplasia (BPD) & 2 & 129 & Risk ratio $(\mathrm{M}-\mathrm{H}$, Fixed, 95\% CI) & $1.65[0.85,3.22]$ \\
\hline Mortality before discharge & 1 & 103 & Risk ratio $(\mathrm{M}-\mathrm{H}$, Fixed, 95\% CI) & Not estimable \\
\hline Serious adverse events & 2 & 129 & Risk ratio (M-H, Fixed, 95\% CI) & Not estimable \\
\hline
\end{tabular}




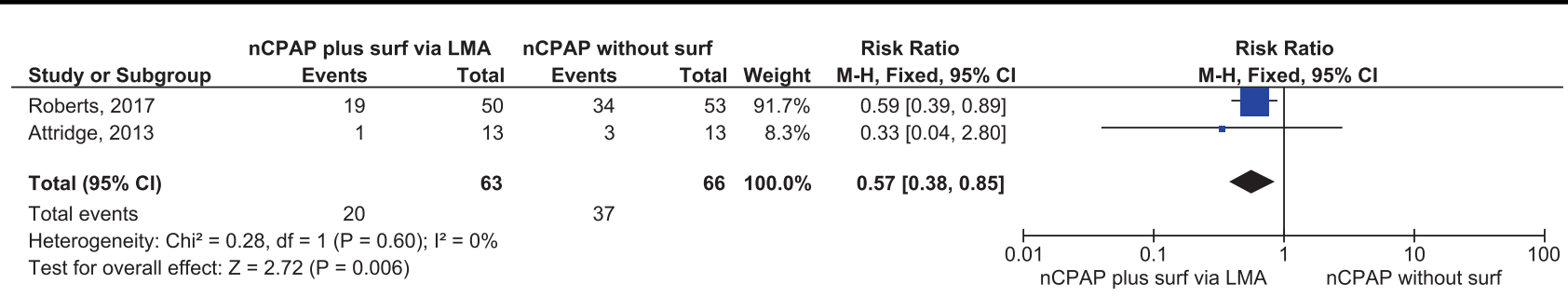

Fig. 4 Need for intubation and/or mechanical ventilation: nCPAP plus surfactant via LMA vs. nCPAP without surfactant (forest plot)

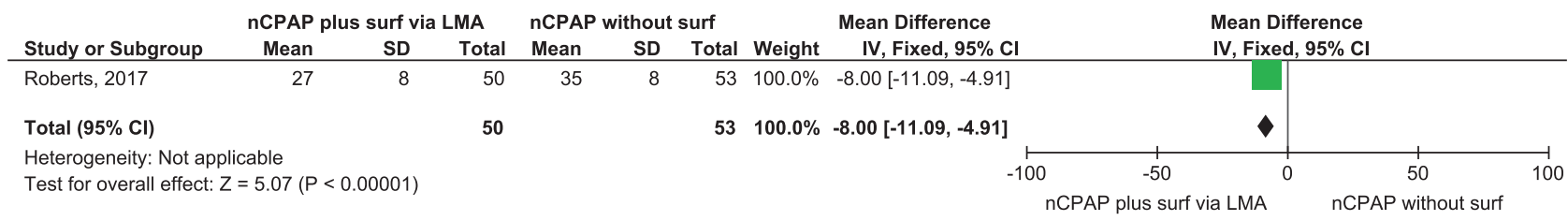

Fig. 5 Oxygen supplementation $(\mathrm{FiO} 2)$ at $1-6 \mathrm{~h}$ after surfactant treatment: nCPAP plus surfactant via LMA vs. nCPAP without surfactant (forest plot)

Table 3 Data and analysis: nCPAP plus surfactant delivery via LMA vs nCPAP plus surfactant delivery via ETT

\begin{tabular}{lllll}
\hline Outcome & No. of studies & Participants & Statistical method & Effect estimate \\
\hline Primary outcomes & & & & \\
Need for intubation and/or mechanical ventilation & 3 & 178 & Risk Ratio (M-H, Fixed, 95\% CI) & 0.43 [0.31-061] \\
FiO2 at 1-6h after surfactant administration & 2 & 108 & Mean difference (IV, Fixed, 95\% CI) & 3.10 [0.51, 5.69] \\
FiO2 at 12-24h after surfactant administration & 1 & 60 & Mean difference (IV, Fixed, 95\% CI) & 3.00 [-2.59, 8.59] \\
Secondary outcomes & & & & \\
Number of surfactant doses & 1 & 60 & Mean difference (IV, Fixed, 95\% CI) & 0.50 [0.08, 0.92] \\
Need $\geq 2$ doses of surfactant & 3 & 178 & Risk Ratio (M-H, Fixed, 95\% CI) & 1.02 [0.44, 2.36] \\
Pneumothorax & 3 & 178 & Risk Ratio (M-H, Fixed, 95\% CI) & 1.63 [0.55, 4.83] \\
Intraventricular hemorrhage (IVH) & 1 & 48 & Risk Ratio (M-H, Fixed, 95\% CI) & 1.41 [0.38, 5.25] \\
Bronchopulmonary dysplasia & 2 & 108 & Risk Ratio (M-H, Fixed, 95\% CI) & 3.05 [0.87, 10.75] \\
Death & 2 & 108 & Risk Ratio (M-H, Fixed, 95\% CI) & 0.17 [0.01, 3.37] \\
Length of hospital stay & 2 & 108 & Mean Difference (IV, Fixed, 95\% CI) & -3.88 [-11.06, 3.29] \\
Bradycardia & 1 & 60 & Risk Ratio (M-H, Fixed, 95\% CI) & 0.33 [0.07, 1.52] \\
Surfactant reflux and/or gastric residuals & 2 & 130 & Risk Ratio (M-H, Fixed, 95\% CI) & 1.00 [0.55, 1.82] \\
\hline
\end{tabular}

\begin{tabular}{|c|c|c|c|c|c|c|c|c|c|}
\hline \multirow[b]{2}{*}{ Study or Subgroup } & \multicolumn{2}{|c|}{ nCPAP plus surf via LMA } & \multicolumn{2}{|c|}{ nCPAP plus surf via ETT } & \multirow[b]{2}{*}{ Weight } & \multirow{2}{*}{$\begin{array}{c}\text { Risk Ratio } \\
\text { M-H, Fixed, } 95 \% \mathrm{Cl}\end{array}$} & \multirow{2}{*}{\multicolumn{2}{|c|}{$\begin{array}{c}\text { Risk Ratio } \\
\text { M-H, Fixed, 95\% Cl }\end{array}$}} & \\
\hline & Events & Total & Events & Total & & & & & \\
\hline Barbosa, 2017 & 12 & 26 & 22 & 22 & $51.4 \%$ & $0.47[0.31,0.71]$ & $\square-$ & & \\
\hline Pinheiro, 2016 & 9 & 30 & 23 & 30 & $48.6 \%$ & $0.39[0.22,0.70]$ & - & & \\
\hline Sadeghnia, 2014 & 0 & 35 & 0 & 35 & & Not estimable & & & \\
\hline Total $(95 \% \mathrm{Cl})$ & & 91 & & 87 & $100.0 \%$ & $0.43[0.31,0.61]$ & & & \\
\hline Total events & 21 & & 45 & & & & & & \\
\hline $\begin{array}{l}\text { Heterogeneity: } \mathrm{Chi}^{2}= \\
\text { Test for overall effect: }\end{array}$ & $\begin{array}{l}29, \mathrm{df}=1(\mathrm{P}= \\
=4.72(\mathrm{P}<0.0\end{array}$ & $=0 \%$ & & & & 0.01 & 0.1 & 1 & 100 \\
\hline
\end{tabular}

Fig. 6 Need for intubation and/or mechanical ventilation: nCPAP plus surfactant via LMA vs. nCPAP plus surfactant via intubation (INSURE) (forest plot)

Data on bradycardia was reported only by Pinheiro et al. observing 2 events in the LMA group and 6 in the control group (RR 0.33, 95\% CI 0.07-1.52; participants $=60$ ) [25]. Pinheiro et al. did not report serious adverse events [25], while this information was not available in Barbosa et al. and Sadeghnia et al. [27, 28]. Gastric residuals was reported in Pinheiro et al. (8/30 vs 10/30) and Sadeghnia et al. (8/35 vs 6/35) (RR 1.00, 95\% CI 0.55-1.82; participants $=130)$ $[25,28]$. Barbosa et al. reported that 4 out of $23(17.4 \%)$ patients had gastric contents $>1.5 \mathrm{ml}$ [27]. 


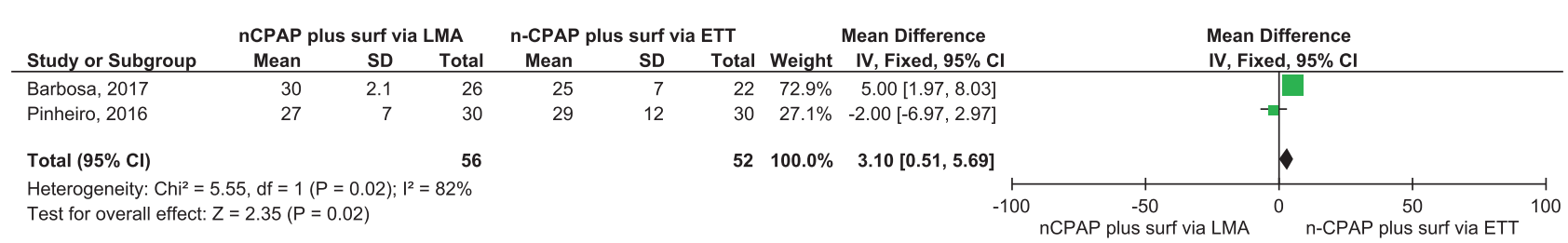

Fig. 7 Oxygen supplementation $(\mathrm{FiO} 2)$ at $1-6 \mathrm{~h}$ after surfactant treatment: nCPAP plus surfactant via LMA vs. nCPAP plus surfactant via endotracheal intubation (INSURE) (forest plot)

\section{Discussion}

Currently, the most common method for administering surfactant to preterm infants is via an endotracheal tube [1, $2,6]$. However, tracheal intubation is an invasive procedure carrying its own risk of complications and requiring skilled personnel $[9,30]$. As a SAD is less invasive and easier to place than an endotracheal tube, it could be considered as the ideal interface for administering surfactant in preterm infants with RDS [17-19].

This study reviews existing literature on RCTs comparing surfactant administration via $\mathrm{SAD}$ versus $\mathrm{nCPAP}$ alone without surfactant (2 studies) [24, 26], or via SAD versus endotracheal administration of surfactant (3 studies) $[25,27,28]$ in preterm infants with non-invasive respiratory support.

Our meta-analysis shows that surfactant delivery via SAD significantly reduces the need for intubation and/or mechanical ventilation when compared with either nCPAP alone or surfactant administration via INSURE. Moreover, surfactant delivery via SAD provides a transient (1-6h) reduction in oxygen requirements when compared with no treatment other than continued CPAP. However, surfactant delivery via SAD is associated with increased oxygen requirements at $1-6 \mathrm{~h}$ when compared with surfactant delivery via INSURE. These findings suggest that SAD technique may replace endotracheal tube in surfactant administration, but surfactant delivery to the lungs via SAD seems less effective than via INSURE. In addition, surfactant delivery via SAD is associated with an increased number of surfactant doses when compared with either nCPAP alone or surfactant administration via INSURE. It is likely that a fraction of surfactant dose did not reach the lungs and was lost in upper airways or stomach when delivered via SAD. This aspect could increase the costs of treatment and should be balanced with reduced need for intubation and mechanical ventilation. Moreover, SAD positioning is easier when compared with endotracheal intubation [17-19], thus settings with limited skills in intubation may benefit from the availability of a SAD.

Surfactant treatment reduces air leak and mortality in preterm infants with RDS [1-6]. This systematic review does not identify any advantage of SAD regarding important clinical outcomes such as pneumothorax, IVH, BPD, mortality, and length of hospital stay. Further studies are needed to evaluate the effect of surfactant administration via SAD on these relevant clinical outcomes.

This systematic review includes studies using different SAD models (classic LMA, ProSeal LMA and I-gel); (Fig. 1) [24-28]. Both classic and ProSeal LMAs are equipped with an inflatable cuff, while I-gel is a cuffless SAD. However, available data do not allow the comparison of different SAD models on surfactant administration. Actually, availability of SAD for neonatal use is restricted to size 1 , which is indicated for newborns weighting $2-5 \mathrm{~kg}$, while a smaller size should be introduced for preterm newborns. In the included studies, investigators were $100 \%$ successful inserting the SAD (despite the number of attempts were not reported).

Regarding the applicability of evidence, the use of SAD does not consistently show any short or long-term advantages over current techniques. Some relevant questions still remain unanswered, including whether SAD may replace available approaches for surfactant administration (INSURE or LISA or MIST) and which is the most adequate SAD for surfactant delivery among available ones (i.e., preterm infants may need smaller sizes). Moreover, it is unclear which is the best procedure for SAD and catheter placement, whether the sedation is needed for the procedure and which respiratory support (CPAP or PPV) should be used for surfactant delivery. Overall, there is lack of information regarding the incidence of side effects (i.e., bradycardia, hypoxia, blood pressure fluctuations) using SAD compared to available approaches (INSURE, LISA, MIST), as well as the effects of surfactant administration via SAD on short and long-term clinical outcomes.

Compared to the available literature regarding this topic, a Cochrane review published in 2011 suggested that surfactant administration in preterm infants with established RDS might have a short-term effect in reducing oxygen requirements [31]. Our review includes five studies and compares surfactant administration via SAD vs. nasal CPAP alone or INSURE, while the 2011 Cochrane review included only one study comparing surfactant administration via $\mathrm{SAD}$ vs. nasal CPAP alone. Six small observational studies including 22 patients (range 1-8 patients/study) were excluded from this review [15, 16, 32-35]. All the studies, except one [33], reported a significant improvement in 
oxygenation after surfactant administration via LMA without side effects. In one study including 4 patients with RDS [33], surfactant was administered via a catheter passed 'blindly' through LMA into the trachea. Oxygenation did not improve from baseline to $3 \mathrm{~h}$ after the treatment suggesting that a large portion of the surfactant did not reach the lungs. The authors assumed that the diminished response was due to the wrong positioning of the catheter tip. Our results confirm that surfactant delivery via LMA is feasible, but many aspects of the entire procedure need to be further investigated.

The findings of our study should be interpreted within its limitations. First, all included studies enrolled a limited number of newborns. Second, included studies used three different models of SAD, different procedures, and different control groups, which may have affected the study results. Third, the quality of the evidence supporting the pooled outcome measures was very low, according to the GRADE guidelines, due to limitation in the study design, early termination in some studies and imprecision of the estimates (few studies; few events). Results of smaller studies are subject to greater sampling variation, and hence are less precise. Indeed, imprecision is reflected in the confidence interval around the intervention effect estimate from each study and in the weight given to the results of each study included in the metaanalysis.

\section{Conclusion}

In preterm infants with RDS receiving respiratory support, surfactant administration via SAD reduces the need for intubation/mechanical ventilation and increases the amount of required surfactant. Short-term oxygen requirements decrease with SAD compared to nCPAP alone, but increase compared to INSURE approach. This systematic review does not identify any advantage of SAD regarding important clinical outcomes. Overall, available literature includes few small, poor-quality studies that prevent drawing strong conclusions.

Additional studies on surfactant administration via SAD should provide more information on the procedure including model and size of SAD, technique of SAD and catheter placement (i.e., blind or under direct vision), use of local or systemic anesthetic drugs, respiratory strategy (NCPAP or PPV) during surfactant delivery. Future RCTs should aim to enroll a larger number of infants and should assess clinically relevant outcomes. The development of a smaller size of SAD for very low birth weight infants should be considered. Surfactant treatment via SAD should be limited to clinical trials.
Acknowledgements We thank Dr. Stubbs Brendon, Institute of Psychiatry, Psychology and Neuroscience, Kings College London, UK for helping in literature search. We are grateful to the authors of 5 included trials for providing additional study information for this systematic review.

Author contributions MGC analyzed the data, contributed to interpret the results and draft the manuscript, and approved the final manuscript as submitted. NV conceived and designed the study, contributed to the data analysis, and approved the final manuscript as submitted. FC contributed to interpret the results and draft the manuscript, and approved the final manuscript as submitted. PC and MM conducted the literature search, extracted key data from the included articles, critically revised the manuscript, and approved the final manuscript as submitted. DT conceived and designed the study, drafted the manuscript, and approved the final manuscript as submitted. All the Authors are responsible for the accuracy and the integrity of the data.

\section{Compliance with ethical standards}

Conflict of interest The authors declare that they have no conflict of interest.

\section{References}

1. Owen LS, Manley BJ, Davis PG, Doyle LW. The evolution of modern respiratory care for preterm infants. Lancet. 2017;389: $1649-59$.

2. Warren JB, Anderson JM. Core concepts: respiratory distress syndrome. NeoReviews. 2009;10:351-61.

3. Seger N, Soll R. Animal derived surfactant extract for treatment of respiratory distress syndrome. Cochrane Database Syst Rev. 2009;2:CD007836.

4. Soll RF. Synthetic surfactant for respiratory distress syndrome in preterm infants. Cochrane Database Syst Rev. 2000;CD001149.

5. Bahadue FL, Soll R. Early versus delayed selective surfactant treatment for neonatal respiratory distress syndrome. Cochrane Database Syst Rev. 2012;11:CD001456.

6. Sardesai S, Biniwale M, Wertheimer F, Garingo A, Ramanathan R. Evolution of surfactant therapy for respiratory distress syndrome: past, present, and future. Pediatr Res. 2017;81:240-8.

7. Verder H, Robertson B, Greisen G, Ebbesen F, Albertsen P, Lundstrøm $\mathrm{K}$, et al. Surfactant therapy and nasal continuous positive airway pressure for newborns with respiratory distress syndrome. Danish-Swedish Multicenter Study Group. N Engl J Med. 1994;331:1051-5.

8. Carbajal R, Eble B, Anand KJ. Premedication for tracheal intubation in neonates: confusion or controversy? Semin Perinatol. 2007;31:309-17.

9. Marshall TA, Deeder R, Pai S, Berkowitz GP, Austin TL. Physiologic changes associated with endotracheal intubation in preterm infants. Crit Care Med. 1984;12:501-3.

10. Ghanta S, Abdel-Latif ME, Lui K, Ravindranathan H, Awad J, Oei J. Propofol compared with the morphine, atropine, and suxamethonium regimen as induction agents for neonatal endotracheal intubation: a randomized, controlled trial. Pediatrics. 2007;119:e1248-1255.

11. Kribs A, Roll C, Göpel W, Wieg C, Groneck P, Laux R, et al. NINSAPP trial investigators. nonintubated surfactant application vs conventional therapy in extremely preterm infants: a randomized clinical trial. JAMA Pediatr. 2015;169:723-30. 
12. Dargaville PA, Aiyappan A, De Paoli AG, Kuschel CA, Kamlin $\mathrm{CO}$, Carlin JB, et al. Minimally-invasive surfactant therapy in preterm infants on continuous positive airway pressure. Arch Dis Child Fetal Neonatal Ed. 2013;98:F122-6.

13. More K, Sakhuja P, Shah PS. Minimally invasive surfactant administration in preterm infants: a meta-narrative review. JAMA Pediatr. 2014;168:901-8.

14. Oncel MY, Arayici S, Uras N, Alyamac-Dizdar E, Sari FN, Karahan S, et al. Nasal continuous positive airway pressure versus nasal intermittent positive-pressure ventilation within the minimally invasive surfactant therapy approach in preterm infants: a randomised controlled trial. Arch Dis Child Fetal Neonatal Ed. 2016;101:F323-8.

15. Brimacombe J, Gandini D, Keller C. The laryngeal mask airway for administration of surfactant in two neonates with respiratory distress syndrome. Paediatr Anaesth. 2004;14:188-90.

16. Trevisanuto D, Grazzina N, Ferrarese P, Micaglio M, Verghese C, Zanardo V. Laryngeal mask airway used as a delivery conduit for the administration of surfactant to preterm infants with respiratory distress syndrome. Biol Neonate. 2005;87:217-20.

17. Hohlrieder M, Brimacombe J, von Goedecke A, Keller C. Postoperative nausea, vomiting, airway morbidity, and analgesic requirements are lower for the ProSeal laryngeal mask airway than the tracheal tube in females undergoing breast and gynaecological surgery. Br J Anaesth. 2007;99:576-80.

18. van Esch BF, Stegeman I, Smit AL. Comparison of laryngeal mask airway vs tracheal intubation: a systematic review on airway complications. J Clin Anesth. 2017;36:142-50.

19. Trevisanuto D, Micaglio M, Ferrarese P, Zanardo V. The laryngeal mask airway: potential applications in neonates. Arch Dis Child Fetal Neonatal Ed. 2004;89:F485-9.

20. Higgins JP, Altman DG, Gotzsche PC, Juni P, Moher D, Oxman $\mathrm{AD}$, et al. Cochrane Bias Methods G, Cochrane Statistical Methods G. The Cochrane Collaboration's tool for assessing risk of bias in randomised trials. BMJ. 2011;343:d5928.

21. Schünemann H, Brozek J, Guyatt G, Oxman A (eds). GRADE Working Group. GRADE handbook for grading quality of evidence and strength of recommendations. https://gdt.gradepro.org/a pp/handbook/handbook.html. Accessed October 2013.

22. GRADE Working Group, McMaster University. GRADEpro GDT Version. Hamilton, ON: GRADE Working Group, McMaster University; 2014.
23. GRADEpro. GDT. https://gradepro.org. Accessed 8 April 2018.

24. Roberts KD, Brown R, Lampland AL, Leone TA, Rudser KD, Finer NN, et al. Laryngeal mask airway for surfactant administration in neonates: a randomized, controlled trial. J Pediatr. 2018;193:40-46.e1.

25. Pinheiro JM, Santana-Rivas Q, Pezzano C. Randomized trial of laryngeal mask airway versus endotracheal intubation for surfactant delivery. J Perinatol. 2016;36:196-201.

26. Attridge JT, Stewart C, Stukenborg GJ, Kattwinkel J. Administration of rescue surfactant by laryngeal mask airway: lessons from a pilot trial. Am J Perinatol. 2013;30:201-6.

27. Barbosa RF, ESAC Simoes, Silva. YP. A randomized controlled trial of the laryngeal mask airway for surfactant administration in neonates. J Pediatr. 2017;93:343-50.

28. Sadeghnia A, Tanhaei M, Mohammadizadeh M, Nemati M. A comparison of surfactant administration through i-gel and ETtube in the treatment of respiratory distress syndrome in newborns weighing more than $2000 \mathrm{~g}$. Adv Biomed Res. 2014;3:1-6.

29. Review Manager (RevMan) Version 5.3 [Computer program]. Copenhagen: The Nordic Cochrane Centre, The Cochrane Collaboration; 2014. P.

30. Doglioni N, Cavallin F, Zanardo V, Trevisanuto D. Intubation training in neonatal patients: a review of one trainee's first 150 procedures. J Matern Fetal Neonatal Med. 2012;25:1302-4.

31. Abdel-Latif ME, Osborn DA. Laryngeal mask airway surfactant administration for prevention of morbidity and mortality in preterm infants with or at risk of respiratory distress syndrome. Cochrane Database Syst Rev. 2011;CD008309.

32. Barbosa RF, Marcatto Jde O, Silva AC, Silva YP. ProSealTM laryngeal mask airway for surfactant administration in the treatment of respiratory distress syndrome in a premature infant. Rev Bras Ter Intensiv. 2012;24:207-10.

33. Trevisanuto D, Doglioni N, Mario F, Micaglio M, Parotto M, Zanardo V. Drug administration via the laryngeal mask airway. Resuscitation. 2006;71:263-4.

34. Micaglio M, Zanardo V, Ori C, Parotto M, Doglioni N, Trevisanuto $\mathrm{D}$, et al. for surfactant administration. Paediatr Anaesth. 2008;18:91-92.

35. Vannozzi CiantelliM, Moscuzza F, Scaramuzzo RT, Panizza D, Sigali E, et al. Catheter and Laryngeal mask endotracheal surfactant therapy: the CALMEST approach as a novel MIST technique. J Matern Fetal Neonatal Med. 2017;30:2375-7. 